Article

\title{
Structural Colors Based on Amorphous Arrays Comprised Solely of Silica Particles
}

\author{
Dae-San Choi, Ju-Hwan Choi and Chang-Yull Lee * \\ Department of Aerospace Engineering, Chosun University, Gwangju 61452, Korea; dschoi@ssml.re.kr (D.-S.C.); \\ jhchoi@ssml.re.kr (J.-H.C.) \\ * Correspondence: cylee@chosun.ac.kr; Tel.: +82-62-230-7955
}

Received: 30 November 2019; Accepted: 2 January 2020; Published: 6 January 2020

\begin{abstract}
In this study, structural colors were fabricated by producing an amorphous array with atypical silica particles. The colors were controlled by an array of silica particles with different sizes. In previous research, the process required inducement of the amorphous array, which was complex. Meanwhile, in this paper, we proposed a simple one-step process. First, spherical silica nanoparticles were synthesized using the sol-gel process of the Stöber method. Atypical silica particles that induced an amorphous array were produced by adding a small amount of phenol-formaldehyde resin. Subsequently, the colloidal silica was converted to a powder using a convection oven. The characteristics of the synthesized silica particles were confirmed using a scanning electron microscope (SEM). All the synthesized silica powders obtained structural colors. Finally, the silica powders were dispersed in deionized (DI) water and coated on a glass slide. We confirmed that the silica particles showed different structural colors depending on the size of the particles. We also found that the color was highly independent of the viewing angle.
\end{abstract}

Keywords: phenol-formaldehyde resin; photonic glass; silica particle; structural color

\section{Introduction}

Generally, the color of a substance is determined by its dyestuff and pigment. However, the colors of these chemicals can degrade after prolonged exposure to ultraviolet radiation, which can lead to environmental pollution and product cost issues. Structural colors are gaining attention as a way to solve the problems above. The structural color is composed of micro-structures with intervals at the wavelengths level of light. This structure causes a constructive interference effect for a specific wavelength of light and destructive interference for the other light wavelengths [1-5]. Additionally, structural color is generated by the Bragg's diffraction condition, upon occasion, producing a rainbow of colors [6]. Structural colors have physical properties that can overcome the limitations of color expression, which occur when conventional chemically colored artificial pigments are used. Moreover, it has the advantages of environmental friendliness, productivity, low cost, and high sustainability [7-10].

Structural color expression in colloidal particles is classified into two types: a regular array and an irregular array of particles. Structural color with a regular array of particles changes color depending on the viewing angle. This material is called a photonic crystal. The structural color associated with an irregular array of particles is less dependent on the viewing angle. This substance is called a photonic glass [11-13]. In particular, photonic glasses without angle dependency have the same optical characteristics in all directions. These characteristics can increase the energy efficiency of solar cells and can be used in paint or cosmetic materials with super-hydrophobicity and high sustainability.

Over the past two decades, research on photonic glasses has been actively pursued. Forster et al. [14] fabricated structural color films on glass coverslips using spin-cast spherical polystyrene particles with average diameters of $226 \mathrm{~nm}$ and $271 \mathrm{~nm}$. The produced film did not change color with the 
change in the viewing angle and it appeared blue-green. Additionally, spherical polystyrene particles and carbon black were mixed to express structural color intuitively, where the carbon black content controlled color saturation. Similarly, in research on carbon particles, Takeoka et al. [15] attempted to induce an amorphous array of micrometer-sized spherical silica particles by rapid evaporation of the solvent. However, iridescence appeared due to the crystalline arrays. To solve this problem, the authors induced an amorphous array by mixing silica particles and carbon black. This mixture resulted in the fabrication of structural color without angle dependency, with different colors arising depending on the size of the silica particles. In another case, Teshima et al. [16] created structural colors by producing monodispersed spherical aggregates using micro-flow-focusing devices. The various colors produced depended on the concentration of colloidal silica, the size of the silica particles, and the amount of magnetite colloidal particles used. In recent years, Zhang et al. [17] finely controlled the ratio by mixing spherical polystyrene particles and cuttlefish ink particles to obtain various high purity colors. Additionally, Gokhan et al. [18] used a centrifuge to replace the solvent of the colloidal particles with ethanol. They then dispersed it on a substrate to form colloidal aggregates with non-iridescent properties.

However, the evaporation process is complicated when a variety of structural colors are fabricated under various conditions. Therefore, this calls for a method that can simplify the formation of a photonic glass without using an additional process or particles. In this paper, a simple method for fabricating structural colors using the sol-gel process with low angle-dependence was proposed, which did not require the addition of other particles. Atypical silica was prepared by adding phenol-formaldehyde resin. Silica powders appeared in a blue, green, and red color depending on the particle size, and the colors of the powders were independent of various viewing angles. Moreover, photonic glass was obtained by evaporating the silica solution redispersed in DI water on a glass slide. Both spherical silica nanoparticles and atypical silica particles inducing an amorphous arrangement were fabricated through the one-step synthesis process. Structural color arising solely from silica particles has different colors depending on the sizes of the silica particles, and it is highly independent of viewing angle change.

\section{Experimental Setup}

\subsection{Measurements and Materials}

The arrangement and particle shape of the silica particles were monitored using scanning electron microscopy (SEM, S-4800, Hitachi, Tokyo, Japan). The product synthesized through the sol-gel process was examined using x-ray diffraction (XRD, X'pert Pro MRD, PANalytical, Almelo, The Netherlands). The size of the silica particles was measured using a nano-micro particle size analyzer (PSA, Scatteroscope-I, Qudix, Seoul, Korea). The reagents used for silica synthesis were deionized (DI) water (extra pure grade, Duksan, Gyeonggi-do, Korea), ethanol (EtOH, 95\%, Duksan), ammonia water (28.0-30.0\%, Samchun, Seoul, Korea), tetraethyl orthosilicate (TEOS, 95\%, Samchun), and phenol-formaldehyde resin. The phenol-formaldehyde resin was synthesized from phenol $(99 \%$, Samchun) and formaldehyde (35\%, Samchun) in a ratio of 1:1.5 [19].

\subsection{Synthesis of Spherical Silica Nanoparticles}

Figure 1 shows the manufacturing process for the silica particles. The phenol-formaldehyde resin was dispersed ultrasonically in tetraethyl orthosilicate (TEOS) for $60 \mathrm{~min}$. Ethanol and DI water for the hydrolysis and condensation reactions were added to a round bottom flask and stirred at $600 \mathrm{rpm}$ for $60 \mathrm{~min}$. The phenol-formaldehyde resin/TEOS dispersion was injected into the ethanol/DI water solution and then stirred at $600 \mathrm{rpm}$ for $60 \mathrm{~min}$. Next, ammonia water was injected and stirred at $600 \mathrm{rpm}$ for $120 \mathrm{~min}$. The resulting solution was dried at $90{ }^{\circ} \mathrm{C}$ in a convection oven. Finally, the agglomerates of dried silica were ground to a powder. The content of the phenol-formaldehyde resin was changed to control the particle size of the silica. Three conditions were tested using $0.027 \mathrm{~g}$, $0.030 \mathrm{~g}$, and $0.033 \mathrm{~g}$ of the phenol-formaldehyde resin. Through this method, we prepared spherical 
silica nanoparticles. Additionally, spherical silica nanoparticles were prepared for comparison without injecting the phenol-formaldehyde resin. Table 1 shows the amounts of the reagents used in the synthesis of the spherical silica nanoparticles.

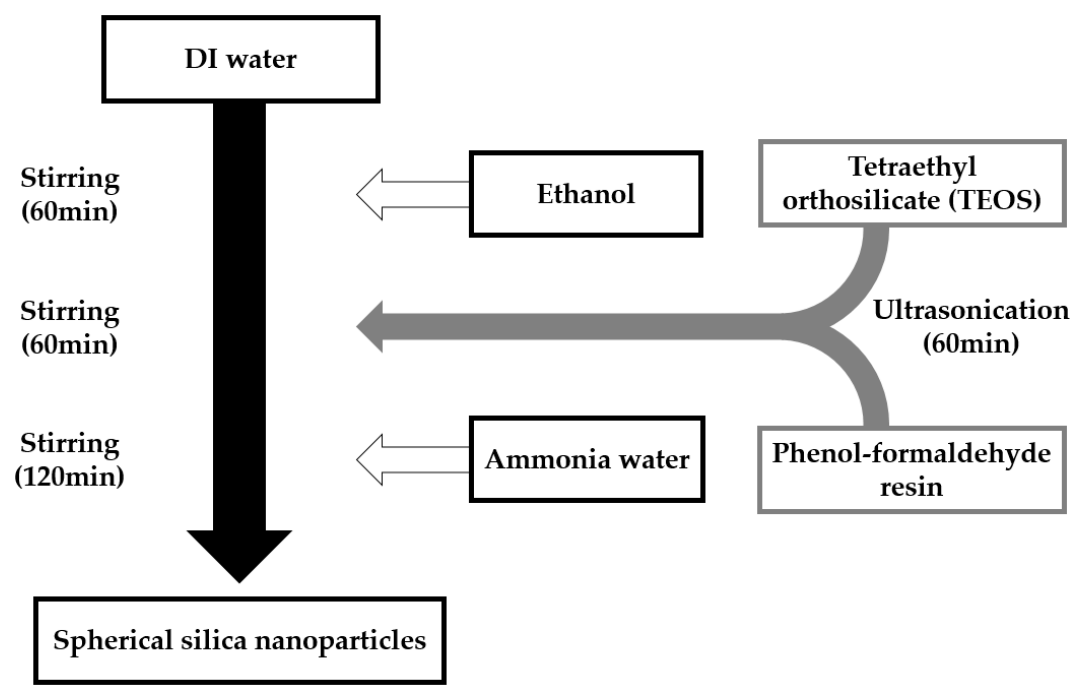

Figure 1. The manufacturing process for silica nanoparticles.

Table 1. Reagents used in the synthesis of spherical silica nanoparticles.

\begin{tabular}{cccccc}
\hline Sample & $\begin{array}{c}\text { Phenol-Formaldehyde } \\
\text { Resin }[\mathbf{g}]\end{array}$ & $\begin{array}{c}\text { TEOS } \\
{[\mathbf{m L}]}\end{array}$ & $\begin{array}{c}\text { Ethanol } \\
{[\mathbf{m L}]}\end{array}$ & $\begin{array}{c}\text { DI Water } \\
{[\mathbf{m L}]}\end{array}$ & $\begin{array}{c}\text { Ammonia } \\
\text { Water }[\mathbf{m L}]\end{array}$ \\
\hline Case 1 & - & 20 & 50 & 50 & 6 \\
Case 2 & 0.027 & 20 & 50 & 50 & 6 \\
Case 3 & 0.030 & 20 & 50 & 50 & 6 \\
Case 4 & 0.033 & 20 & 50 & 50 & 6 \\
\hline
\end{tabular}

\subsection{Coating and Synthesis of Silica Solutions}

Figure 2 shows the coating process for producing structural color using silica solutions. It is impossible to arrange spherical silica nanoparticles in powder form amorphously. Therefore, silica powder was dispersed in a liquid and used in the liquid solution state. In our study, deionized water was used as the solvent. Silica powder was added to the DI water, and the concentration was adjusted to $0.1 \mathrm{~g} / \mathrm{mL}$. The resulting solution containing silica powder was dispersed for $4 \mathrm{~h}$ using an ultrasonic disperser. The silica solutions were then ready for use. The prepared silica solution was coated on an alcohol-washed glass slide $(76 \mathrm{~mm} \times 26 \mathrm{~mm} \times 1 \mathrm{~mm})$ to express the structural colors.
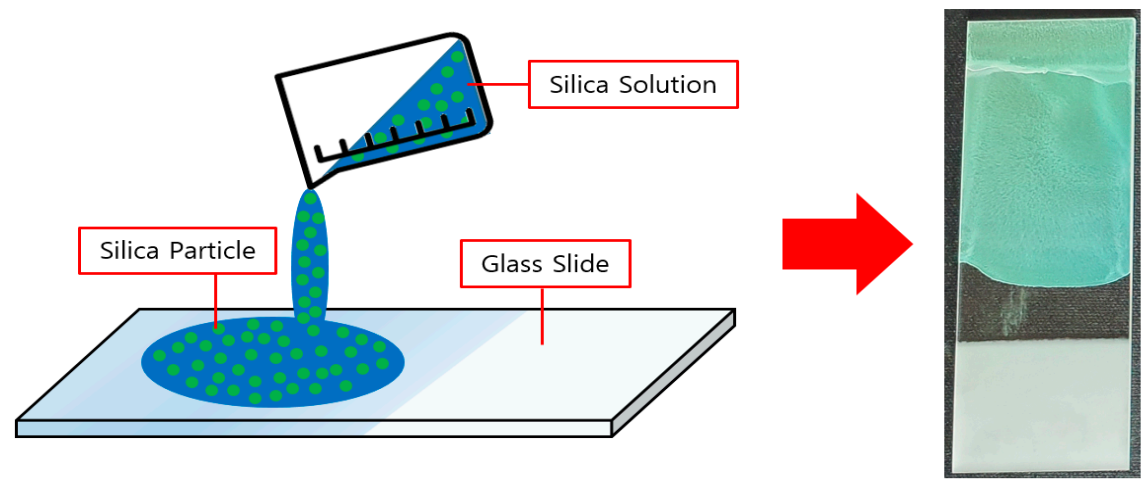

Figure 2. Coating process for the silica solutions. 


\section{Experimental Results and Discussion}

\subsection{Spherical Silica Nanoparticles}

Figure 3 shows the XRD patterns for the products synthesized by the sol-gel process. Each pattern shows a peak in the range of $15-30^{\circ}$. No additional peaks were found in other regions. The trend for these XRD patterns was the same as that for the silica obtained by the sol-gel process reported by Lin et al. [20]. The measured pattern (88\%) was consistent with the $\mathrm{SiO}_{2}$ pattern reported in the crystallography open database (COD, no. 96-901-3492) [21]. We could confirm that the material produced using this method was $\mathrm{SiO}_{2}$.

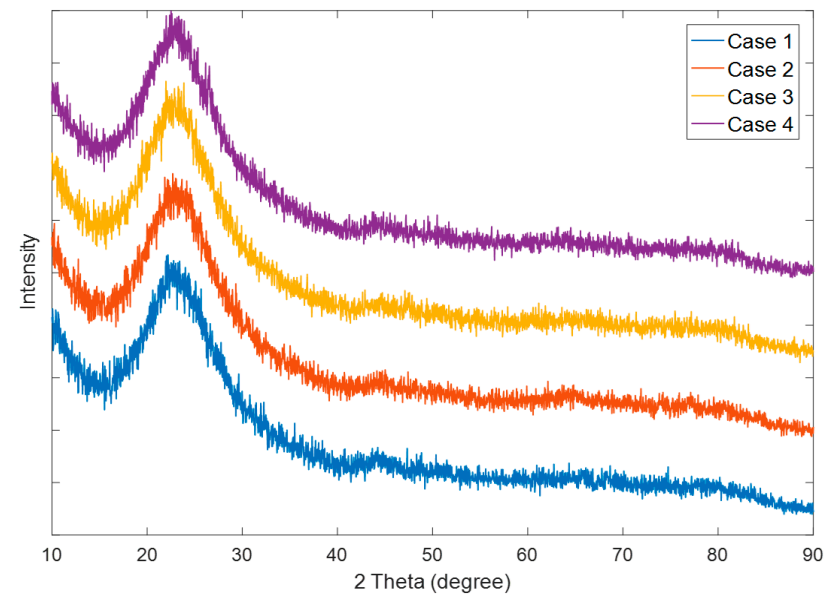

Figure 3. X-ray diffraction (XRD) patterns of the silica powders. Case 1: No added phenol-formaldehyde resin; Case 2: $0.027 \mathrm{~g}$ of added phenol-formaldehyde resin; Case 3: $0.030 \mathrm{~g}$ of added phenol-formaldehyde resin; Case 4: $0.033 \mathrm{~g}$ of added phenol-formaldehyde resin.

Figure 4 presents the SEM images of the silica powder. Figure 4 a shows a silica powder synthesized without the phenol-formaldehyde resin, and the silica particles show uniform spherical shapes and sizes. On the other hand, the silica powders in Figure $4 b-d$ are synthesized with phenol-formaldehyde resin. Spherical or atypical particles were formed. Unlike Figure $4 \mathrm{a}$, some necks are observed between the nanoparticles. This result was due to the phenol-formaldehyde resin.

(a)

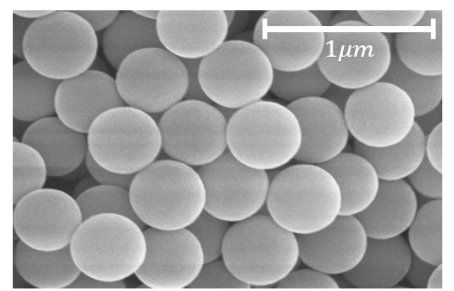

(c)

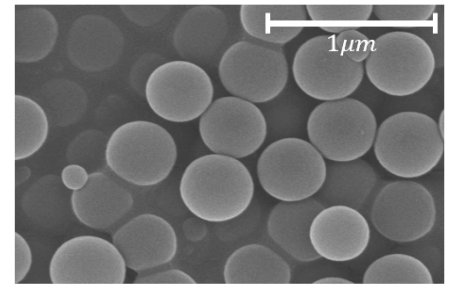

(b)

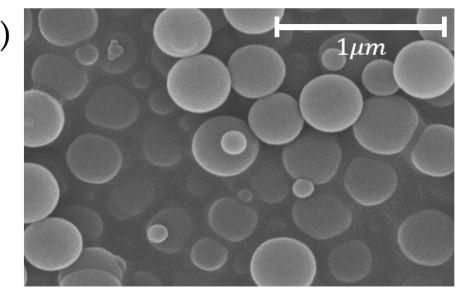

(d)

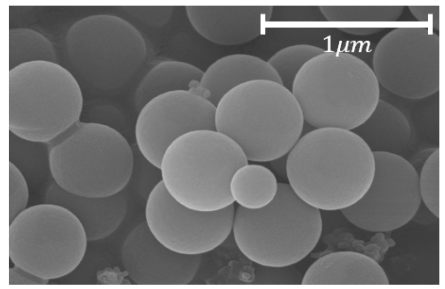

Figure 4. Scanning electron microscopy (SEM) photographs of the silica powders: (a) No added phenol-formaldehyde resin (Case 1); (b) $0.027 \mathrm{~g}$ of added phenol-formaldehyde resin (Case 2); (c) $0.030 \mathrm{~g}$ of added phenol-formaldehyde resin (Case 3); (d) $0.033 \mathrm{~g}$ of added phenol-formaldehyde resin (Case 4). 
Figure 5 indicates the particle size distribution of the manufactured silica powders. Each silica powder was mixed with DI water at a concentration of $0.01 \mathrm{~g} / \mathrm{mL}$ to determine the size of the particles. Subsequently, the mixture was ground in an ultrasonicator for $15 \mathrm{~min}$. Next, the size of the silica particles was measured using a nano-micro particle size analyzer. The average size of the spherical silica nanoparticles without the phenol-formaldehyde resin was $435 \mathrm{~nm}$ (Case 1). Meanwhile, the average size of nanoparticles with the phenol-formaldehyde resin was $359 \mathrm{~nm}, 375 \mathrm{~nm}$, and $502 \mathrm{~nm}$, respectively (Case 2-4). Consequently, the average size of the silica nanoparticles increased with the increasing phenol-formaldehyde resin content.

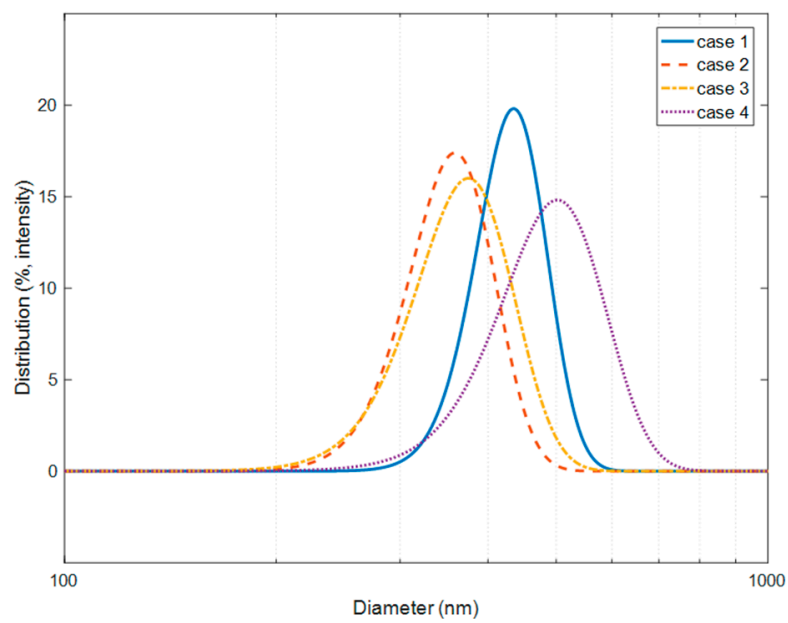

Figure 5. Particle sizes of synthesized silica distributions. No added phenol-formaldehyde resin (Case 1); $0.027 \mathrm{~g}$ of added phenol-formaldehyde resin (Case 2); $0.030 \mathrm{~g}$ of added phenol-formaldehyde resin (Case 3); $0.033 \mathrm{~g}$ of added phenol-formaldehyde resin (Case 4).

Figure 6 shows photographs of the manufactured silica powders. The silica powder of Figure 6a shows an ivory white color typical of silica. The reason for this color was that light scattering off a single particle passes through the surrounding particles, causing multiple scatterings such that the intensity of the scattered light in all wavelengths is the same. Figure $6 \mathrm{~b}-\mathrm{d}$ shows the various structural colors depending on the sizes of the spherical silica nanoparticles. The spherical silica nanoparticles show a blue color at $359 \mathrm{~nm}$, a green color at $375 \mathrm{~nm}$, and red color at $502 \mathrm{~nm}$. Owing to particle aggregation by neck formation and the presence of atypical silica in Figure $4 b-d$, silica powders show structural color. Aggregated particles suppress multiple scatterings and increase the size of the specific wavelength $[14,18]$.
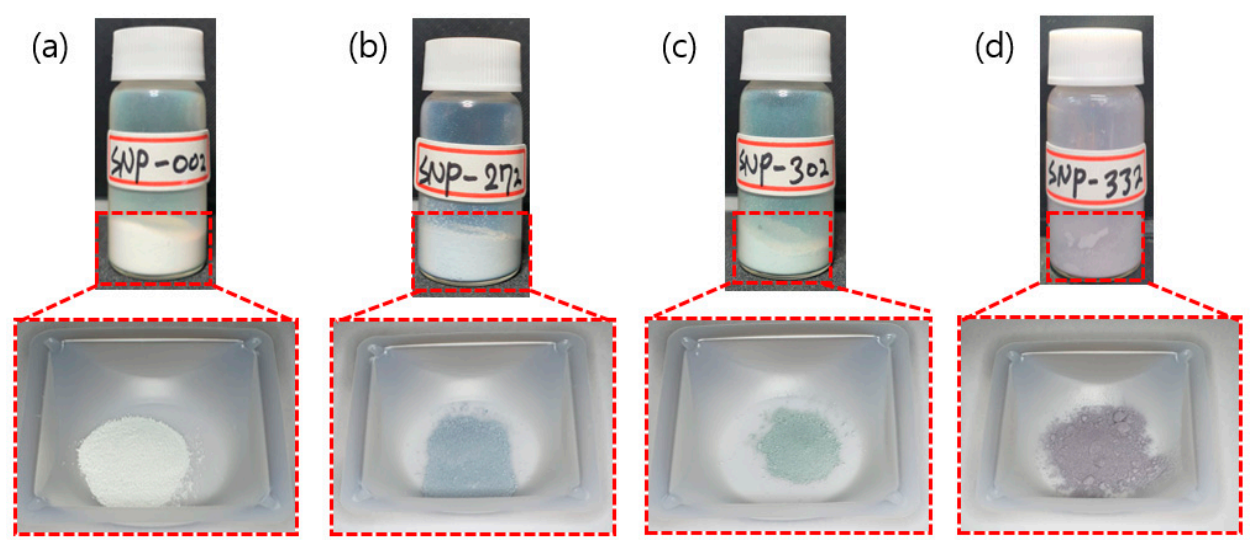

Figure 6. Photographs of the silica powders: (a) No added phenol-formaldehyde resin (Case 1); (b) $0.027 \mathrm{~g}$ of added phenol-formaldehyde resin (Case 2); (c) $0.030 \mathrm{~g}$ of added phenol-formaldehyde resin (Case 3); (d) $0.033 \mathrm{~g}$ of added phenol-formaldehyde resin (Case 4). 


\subsection{Coating of Silica Solutions}

Figure 7 shows the SEM images of the silica solutions coated on a glass slide. Figure 7a shows a crystal lattice structure of a silica solution consisting of homogeneously sized, monodispersed, spherical silica nanoparticles. However, in the case of Figure $7 \mathrm{~b}-\mathrm{d}$, silica particles are arranged in amorphous form instead of a lattice structure. The reason is that the particles of uniform size and shape were arranged in a crystal lattice without disturbing other particles during the self-assembly process. Meanwhile, the spherical silica nanoparticles did not form a lattice structure due to the presence of atypical silica.

(a)

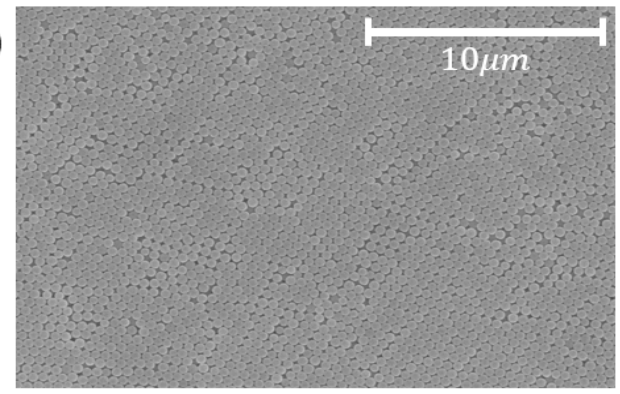

(c)

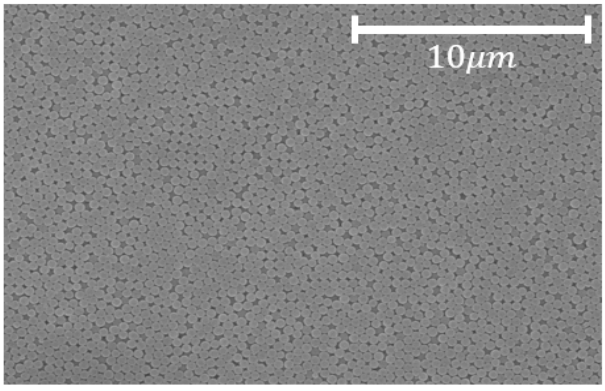

(b)

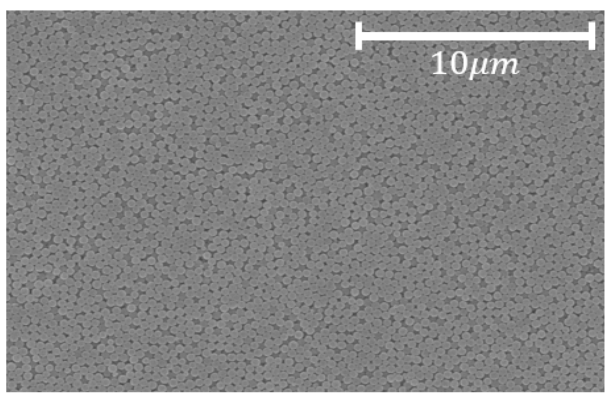

(d)

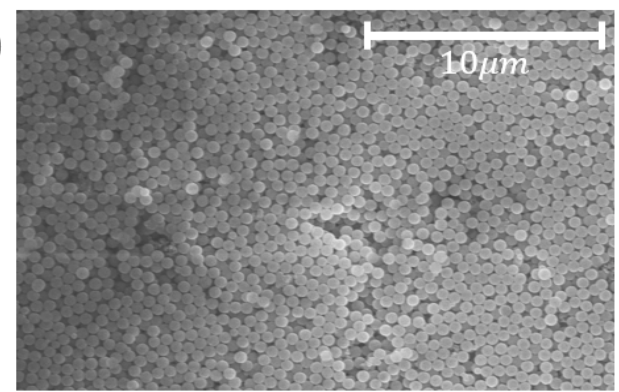

Figure 7. SEM photographs of the silica solution coatings: (a) No added phenol-formaldehyde resin (Case 1); (b) $0.027 \mathrm{~g}$ of added phenol-formaldehyde resin (Case 2); (c) $0.030 \mathrm{~g}$ of added phenol-formaldehyde resin (Case 3); (d) $0.033 \mathrm{~g}$ of added phenol-formaldehyde resin (Case 4).

Figure 8 shows photographs of the coated silica solutions at three viewing angles and photographs of spherical silica nanoparticles rubbed on a black substrate. Figure 8 a shows that the coated silica solution changes color to blue, green, and red at viewing angles of $90^{\circ}, 60^{\circ}$, and $40^{\circ}$. These changes occurred because the crystal lattice structure in case 1 satisfied the Bragg's equation (Equation (1) [11,22]).

$$
\lambda=2 D \sqrt{\frac{2}{3}\left(n_{\left.e f f^{2}-\sin ^{2} \theta\right)}\right.}
$$

where $D, \lambda, \theta$, and $n_{\text {eff }}$ denote the size of the spherical silica nanoparticle, the reflection wavelength, the angle of light incidence, and the effective refractive index, respectively. As shown in Figure 4a, the average size of the spherical silica nanoparticles is $435 \mathrm{~nm}$, and the effective refractive index is 1.35 [18]. The sizes of the particles were calculated theoretically from $438 \mathrm{~nm}$ to $704 \mathrm{~nm}$ with colors, as shown in Figure 8a. In contrast, in Figure $8 b-d$, the silica solutions coated on glass slides show a different color depending on the size of the spherical silica nanoparticles, and the same color as the silica powder is observed. Furthermore, the colors did not change with the change in the viewing angle. These results occurred because spherical silica nanoparticles did not form a crystal lattice structure, but formed an amorphous array due to particle aggregation, as shown in Figure $7 \mathrm{~b}-\mathrm{d}$. That is, atypical silica particles interfered with the crystal arrangement of spherical silica nanoparticles. This amorphous array was similar to the arrangement induced by the addition of carbon black particles, and similar 
results were reported in Reference [14]. Figure 8e indicates that various colored membranes comprise solely of spherical silica nanoparticles. The average sizes of the particles were $300 \mathrm{~nm}, 400 \mathrm{~nm}$, and $500 \mathrm{~nm}$ (from the left in the figure). It is confirmed that the color appeared differently according to the size of the spherical silica nanoparticles. The color of the silica solution coated in this study and the membrane color composed only of spherical silica nanoparticles showed similar trends to Reference [15].

(a)
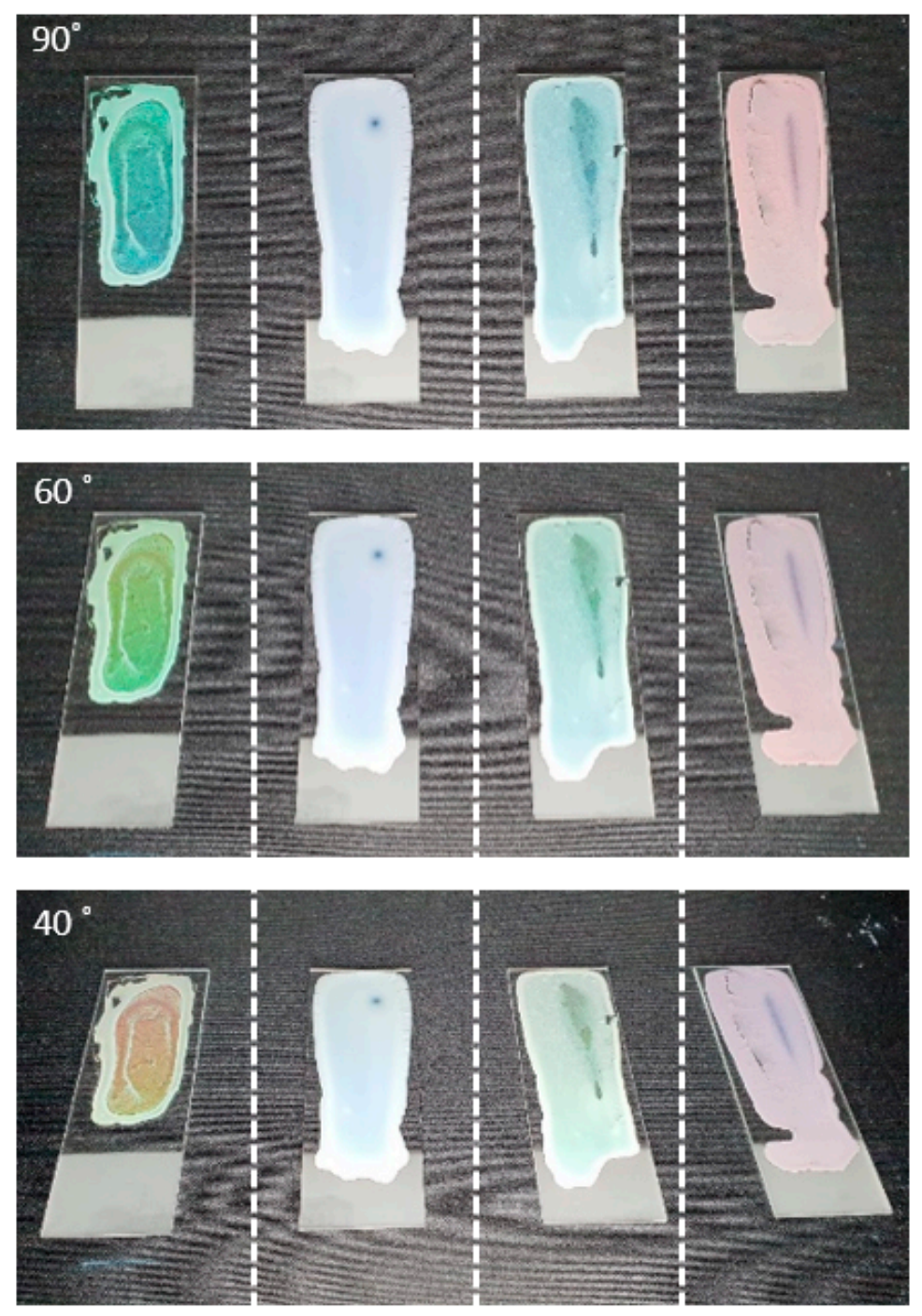

(e)
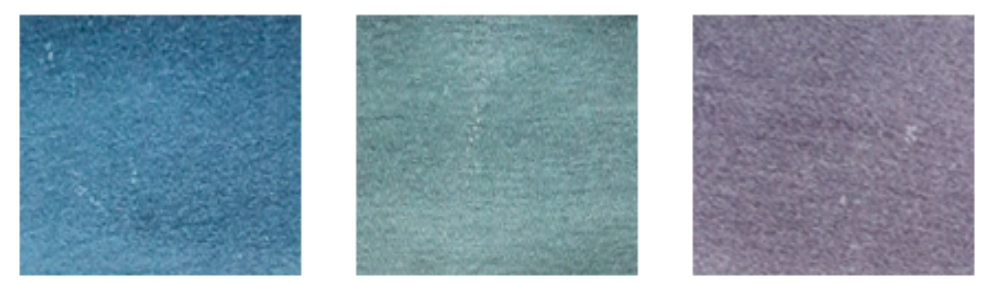

Figure 8. Photographs of the silica solution coatings under three viewing angles: (a) Case 1 (various colors depending on angles); (b) Case 2 (blue); (c) Case 3 (green); (d) Case 4 (red); (e) Colored membranes consist of solely of spherical silica particles. 


\section{Conclusions}

The process of structural color fabrication is challenging, owing to the complicated specific conditions required. Thus, a simple fabricating method with low angle-dependence was proposed without using additional processes or particles. Atypical silica particles and spherical silica nanoparticles were synthesized simultaneously through a one-step process, and structural colors were produced by inducing an amorphous array. The one-step process used the sol-gel process in which TEOS and a small amount of phenol-formaldehyde were mixed before injecting the silica precursor. Subsequently, spherical silica nanoparticles and atypical silica particles were produced. Moreover, the size of the synthesized spherical silica nanoparticles increased proportionately with the amount of phenol-formaldehyde resin added. Similarly, the size of the atypical silica particles also increased. Atypical silica particles form necks between spherical silica nanoparticles and inhibit the formation of a crystal array. Consequently, silica powder shows a different color depending on the size of the spherical silica nanoparticles. On the contrary, silica powder without atypical silica particle appears ivory white. Synthesized silica powder was dispersed in DI water to obtain a photonic glass. Once the colloidal particles are separated and packed, it is difficult to redistribute the individual particles because the surface energy has already been reduced by aggregation. For this reason, aggregated silica particles were suspended in DI water due to the presence of atypical silica. Therefore, the coated silica solution showed the same color as the silica powder. We confirmed that the coated silica solution induced an amorphous arrangement from the SEM images. The results showed that the simple one-step method for fabricating a photonic glass produced structural colors that were independent of viewing angle.

Author Contributions: D.-S.C. participated in the experiment design, experiment, analysis, and writing. J.-H.C. participated in the experiment. C.-Y.L. participated in the supervision, project administration, and review. All authors have read and agreed to the published version of the manuscript.

Funding: This work was supported by the National Research Foundation of Korea (NRF) grant funded by the Korean Government (MSIT) (2017R1C1B2007217). This research was supported by the Basic Science Research Program through the National Research Foundation of Korea (NRF) funded by the Ministry of Education (2019R1I1 A3A01060180).

Conflicts of Interest: The authors declare no conflict of interest.

\section{References}

1. Kinoshita, S.; Yoshioka, S. Structural colors in nature: The role of regularity and irregularity in the structure. Chem. Phys. Chem. 2005, 6, 1442-1459. [CrossRef] [PubMed]

2. Fudouzi, H. Optical properties caused by periodical array structure with colloidal particles and their applications. Adv. Powder Technol. 2009, 20, 502-508. [CrossRef]

3. Kriegel, I.; Scotognella, F. Indium tin oxide nanoparticle: Air layers for one-dimensional multilayer photonic structures. Appl. Sci. Basel 2019, 9, 2564. [CrossRef]

4. Muller, M.; Zentel, R.; Maka, T.; Romanov, S.G.; Torres, C.M.S. Photonic crystal films with high refractive index contrast. Adv. Mater. 2000, 12, 1499-1503. [CrossRef]

5. Li, Z.Y.; Zhang, Z.Q. Photonic bandgaps in disordered inverse-opal photonic crystals. Adv. Mater. 2001, 13, 433-436. [CrossRef]

6. Liu, K.; Schmedake, T.A.; Tsu, R. A comparative study of colloidal silica spheres: Photonic crystals versus Bragg's law. Phys. Lett. A 2008, 372, 4517-4520. [CrossRef]

7. Kanamori, Y.; Katsube, H.; Furuta, T.; Hasegawa, S.; Hane, K. Design and fabrication of structural color filters with polymer-based guided-mode resonant gratings by nanoimprint lithography. Jpn. J. Appl. Phys. 2009, 48, 06FH04. [CrossRef]

8. Mizeikis, V.; Mijulskas, I.; Tomasiunas, R.; Juodkazis, S.; Matsuo, S.; Misawa, H. Optical characteristics of two-dimensional photonic crystals in anodic aluminum oxide films. Jpn. J. Appl. Phys. 2004, 43, 36-43. [CrossRef]

9. Ko, Y.L.; Tsai, H.P.; Lin, K.Y.; Chen, Y.C.; Yang, H. Reusable macroporous photonic crystal-based ethanol vapor detectors by doctor blade coating. J. Colloid Interface Sci. 2016, 487, 360-369. [CrossRef] 
10. Xiao, F.X.; Pagliaro, M.; Xu, Y.J.; Liu, B. Layer-by-layer assembly of versatile nanoarchitectures with diverse dimensionality: A new perspective for rational construction of multilayer assemblies. Chem. Soc. Rev. 2016, 45, 3088-3121. [CrossRef]

11. Kuo, W.K.; Weng, H.P.; Hsu, J.J. Photonic crystal-based sensors for detecting alcohol concentration. Appl. Sci. 2016, 6, 67. [CrossRef]

12. Yu, Y.; Fang, Z.; Ma, C.; Inoue, H.; Yang, G.; Zheng, S.; Chen, D.; Yang, Z.; Masuno, A.; Orava, J.; et al. Mesoscale engineering of photonic glass for tunable luminescence. NPG Asia Mater. 2016, 8, e318. [CrossRef]

13. Armstrong, E.; O'Dwyer, C. Artificial opal photonic crystals and inverse opal structures-Fundamentals and applications from optics to energy storage. J. Mater. Chem. C 2015, 3, 2109-6143. [CrossRef]

14. Forster, D.J.; Noh, H.; Liew, S.F.; Saranathan, V.; Schreck, C.F.; Yang, L.; Park, J.G.; O.Prum, R.; Mochrie, S.G.J.; O'Hern, C.S.; et al. Biomimetic isotropic nanostructures for structural coloration. Adv. Mater. 2010, 22, 2939-2944. [CrossRef]

15. Takeoka, Y.; Yoshioka, S.; Takano, A.; Arai, S.; Nueangnoraj, K.; Nishhara, H.; Teshima, M.; Ohtsuka, Y.; Seki, T. Production of colored pigments with amorphous arrays of black and white colloidal particles. Angew. Chem. 2013, 125, 7402-7406. [CrossRef]

16. Teshima, M.; Seki, T.; Kawano, R.; Takeuchi, S.; Yoshioka, S.; Takeoka, Y. Preparation of structurally colored, monodisperse spherical assemblies composed of black and white colloidal particles using a micro-flow-focusing device. J. Mater. Chem. C 2014, 3, 769-777. [CrossRef]

17. Zhang, Y.; Dong, B.; Chen, A.; Lui, X.; Shi, L.; Zi, J. Using cuttlefish ink as an additive to produce non-iridescent structural colors of high color visibility. Adv. Mater. 2015, 27, 4719-4724. [CrossRef]

18. Gokhan, T.; Tugrul, G.; Mustafa, G.; Mustafa, M.D. Non-iridescent structural colors from uniform-sized SiO2 colloids. Photonics Nanostruct. 2017, 29, 22-29.

19. Qiao, W.; Li, S.; Guo, G.; Han, S.; Ren, S.; Ma, Y. Synthesis and characterization of phenol-formaldehyde resin using enzymatic hydrolysis lignin. J. Ind. Eng. Chem. 2015, 21, 1417-1422. [CrossRef]

20. Lin, W.; Zheng, J.; Yan, L.; Zhang, X. Sol-gel preparation of self-cleaning SiO2-TiO2/SiO2-TiO2 double-layer antireflective coating for solar glass. Results Phys. 2018, 8, 532-536. [CrossRef]

21. Patel, M.; Mukhopadhyday, I.; Ray, A. Annealing influence over structural and optical properties of sprayed SnS thin films. Opt. Mater. 2013, 35, 1693-1699. [CrossRef]

22. Mayonado, G.; Mian, S.M.; Robbiano, V.; Cacialli, F. Investigation of the bragg-snell law in photonic crystals. In Proceedings of the 2015 Laboratory Instruction Beyond the First Year, College Park, MD, USA, 22-24 July 2015.

(C) 2020 by the authors. Licensee MDPI, Basel, Switzerland. This article is an open access article distributed under the terms and conditions of the Creative Commons Attribution (CC BY) license (http://creativecommons.org/licenses/by/4.0/). 